PNL-SA-24176

ALPHA SPECTRAL ANALYSIS VIA ARTIFICIAL NEURAL NETWORKS

RECEMPE

DEC 27.1094

OSTI
L. J. Kangas
G. L. Troyer $^{(a)}$
S. Hashem
P. E. Keller
R. T. Kouzes

October 1994

Presented at the

Nuclear Science Symposium Conference

October 30 - November 5, 1994

Norfolk, Virginia

Prepared for

the U.S. Department of Energy

under Contract DE-AC06-76RLO 1830

Pacific Northwest Laboratory

Richland, Washington 99352

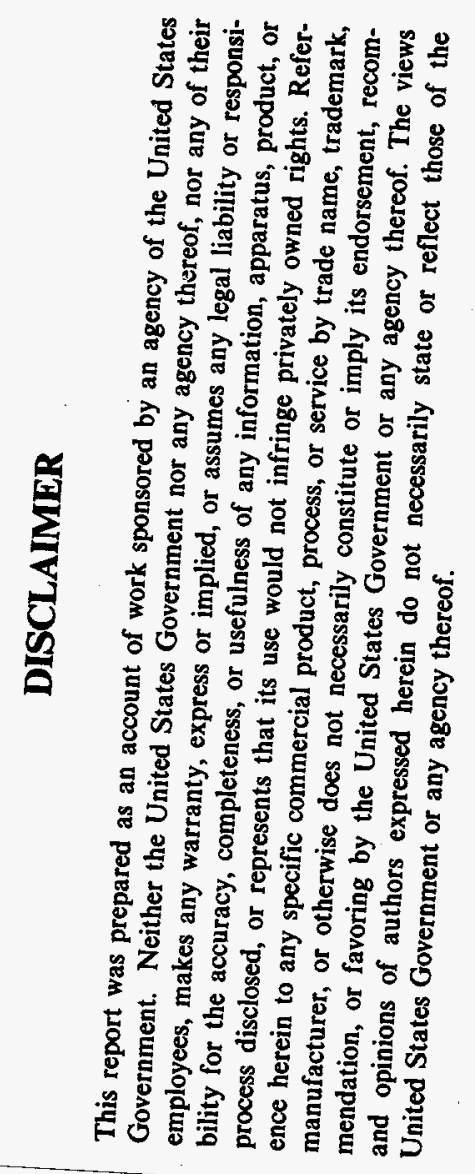

(a) Westinghouse Hanford Company, Richland, Washington 


\section{DISCLAIMER}

Portions of this document may be illegible in electronic image products. Images are produced from the best available original document. 


\title{
Alpha Spectral Analysis via Artificial Neural Networks
}

\author{
Lars J. Kangas, Gary L. Troyer*, Paul E. Keller, Sherif Hashem, and Richard T. Kouzes ${ }^{1}$ \\ Pacific Northwest Laboratory \\ P.O. Box 999, K1-87 \\ Richland, WA 99352 \\ Telephone: (509) 375-3905 \\ E-mail: lj_kangas@pnl.gov \\ *Westinghouse Hanford Company \\ P.O. Box 1970, T6-50 \\ Richland, WA 99352
}

\begin{abstract}
An artificial neural network system that assigns quality factors to alpha particle energy spectra is discussed. The alpha energy spectra are used to detect plutonium contamination in the work environment. The quality factors represent the levels of spectral degradation caused by miscalibration and foreign matter affecting the instruments. A set of spectra was labeled with a quality factor by an expert and used in training the artificial neural network expert system. Our investigation shows that the expert knowledge of alpha spectra quality factors can be transferred to an ANN system.
\end{abstract}

\section{BACKGROUND}

Air quality monitoring for man-made alpha emitting radioisotopes in nuclear processing and storage facilities is performed by measuring collected particulates on air filters. The filters collect fine dust particles including naturally occurring alpha emitters. The filters are observed in real time by alpha spectrometers which create energy spectra. As dust or overmass builds on the filter, the spectral resolution and the ability to differentiate isotopes of interest is compromised.

A desirable goal is to take corrective action before a spectrum degrades beyond usefulness. With significant experience, an operator can determine the usefulness of an energy spectrum by visual inspection. In the case of too much overmass, a simple filter change is performed. In other cases, poor spectral quality may indicate that detector or electronic maintenance is required. Regardless, intervention in the measurement system is required. However, the dynamics of facility operations preclude constant watch of spectral accumulations in real time. Thus, automated early warning based on spectral quality can assure that an isotope of concern is being reliably monitored.

The possibility of using an automated ANN approach to provide real-time inference was proposed as an alternative to more common methods [1]. Many investigators have

\footnotetext{
$\mathbf{1}_{\text {This work was funded by the Environmental Molecular Sciences }}$ Laboratory construction project at Pacific Northwest Laboratory (PNL). PNL is a multiprogram national laboratory operated by Battelle Memorial Institute for the U.S. Department of Energy under Contract DE-AC06-76RLO 1830.
}

attempted to maintain spectral quality through system design or special algorithms [2]. All are limited by the reality of dynamic operations and the potential for unexpected and rapid spectral degradation. Some empirical numerical tests have been performed which are complex ${ }_{1}$ a posteriori exercises. None of these methods have proved attractive for real-time application.

\section{APPROACH}

This investigation was performed on 139 alpha energy spectra received from Westinghouse Hanford Company. The spectra were generated to identify the presence of airborne plutonium in work environments. Minute levels of plutonium are easily lost in the background noise from degraded alpha emitting progeny of radon gas. To be useful for identifying plutonium, these spectra must be of high quality.

ANNs were applied to the problem of assessing the degradation in the quality of the alpha spectra, a task that is presently performed by human experts. Experts use graphical representations of spectra during assessments. The ANN technology was chosen because it has the same capability as experts in analyzing spectral channels in parallel. ANNs have demonstrated their benefits in other applications of spectral analysis [3-6].

The spectra used in this investigation showed various levels of quality degradation due to variables such as calibration and salt accumulation on the filter used in counting the alpha particles. Each spectrum was labeled with a quality factor from 0 to 10 by an expert. The spectra labeled from 0 to 3 are acceptable for determining plutonium levels. The labels in the midrange (4 to 6) are marginally acceptable. High labels, above 6 , indicate poor quality spectra and that the filter needs replacement and/or that the instrument needs calibration. The task for the ANN was to learn to label spectra with quality factors using the knowledge of a human expert. 


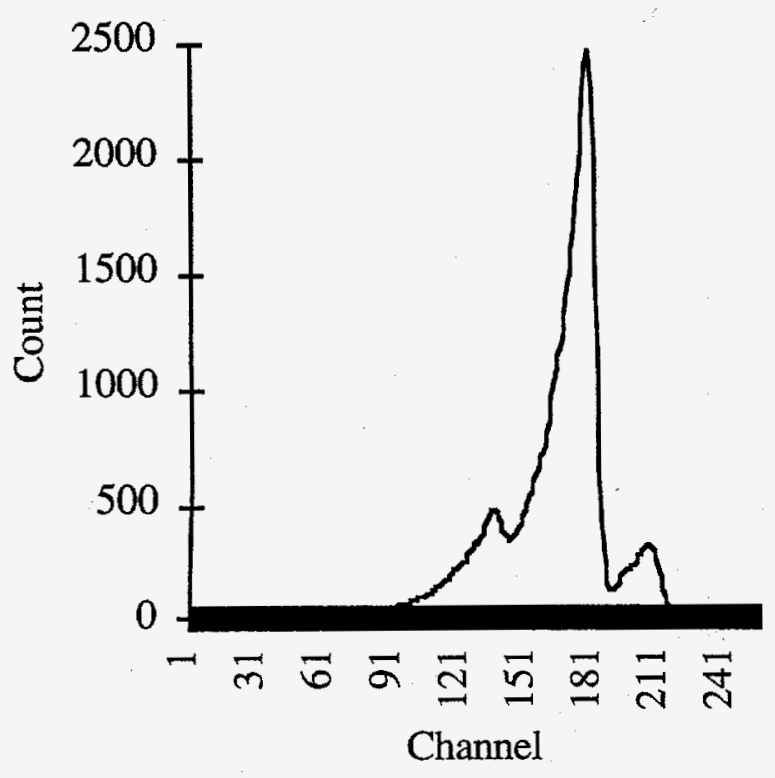

(a)

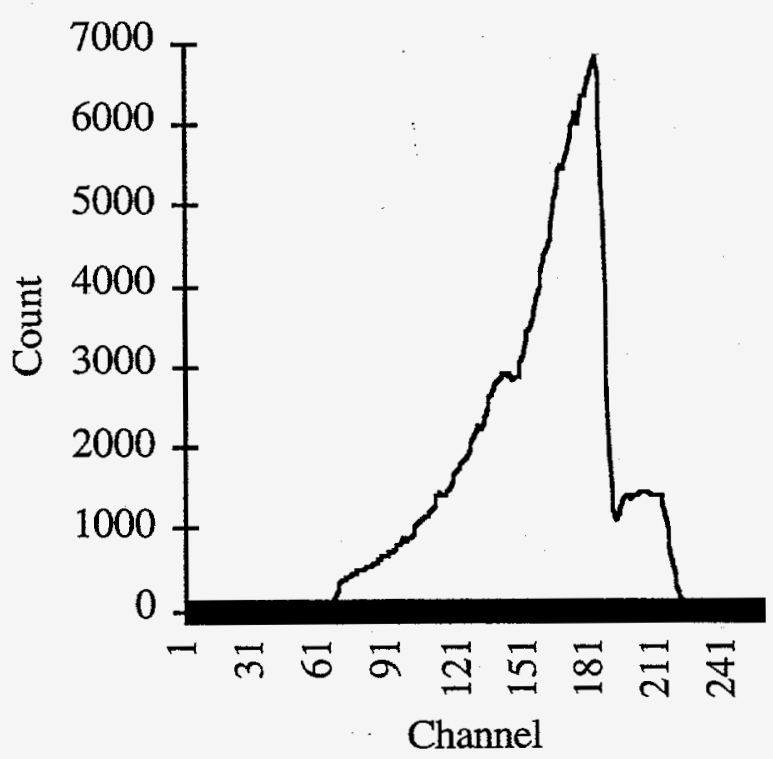

(b)

Fig 1. Alpha spectra. (a) shows an optimal spectra with an expert assessed quality coefficient of 1 . (b) shows a degraded spectra with an expert assessed quality coefficient of 5 . Observe that the differences in the scale of the vertical axis are insignificant. The vertical axis represent energy count and is not analyzed as absolute values.

The original alpha spectra were sampled at 512 channels and reduced to 256 channels during the data collection. It was observed during data analysis that all the spectral information was found between channels 40 and 239. These 200 channels were further processed to reduce the amount of data being fed into the ANN. The 200 channels were reduced into 20 equal sized channels, with each new channel computed as the average of 10 consecutive channels. This reduction in the spectral resolution was deemed appropriate for the relatively few spectra used in this investigation. The channel counts in each spectrum were normalized to the maximum channel count of the spectrum before presentation to the ANN.

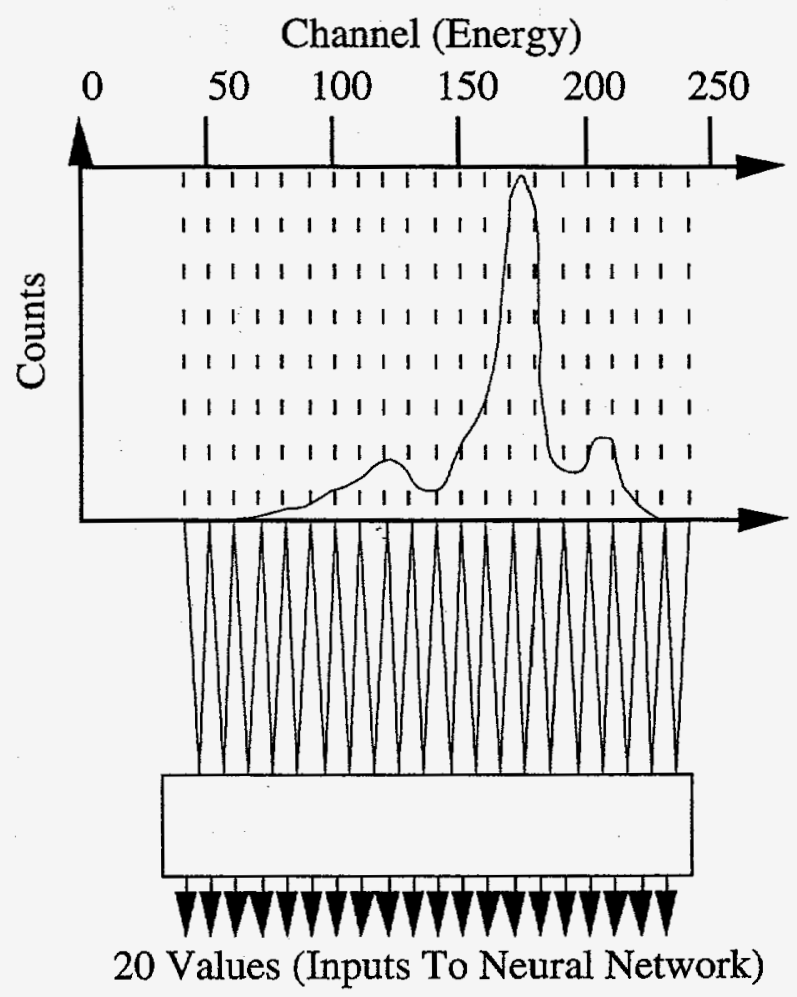

Fig 2. Preprocessing of the 256 spectral channels reduces the number of values to 20 .

The ANN used in this investigation consisted of 20 inputs for the averaged spectral values, one hidden layer with 15 nodes, and one output node for the spectral quality value. The ANNs were trained using the standard backpropagation algorithm with batch mode weight updates. The learning rate and momentum were kept constant at 0.01 and 0.9 , respectively. The training of the ANNs was stopped at 3000 epochs. This ANN configuration and these training variables had been previously determined to yield near optimal performance. 


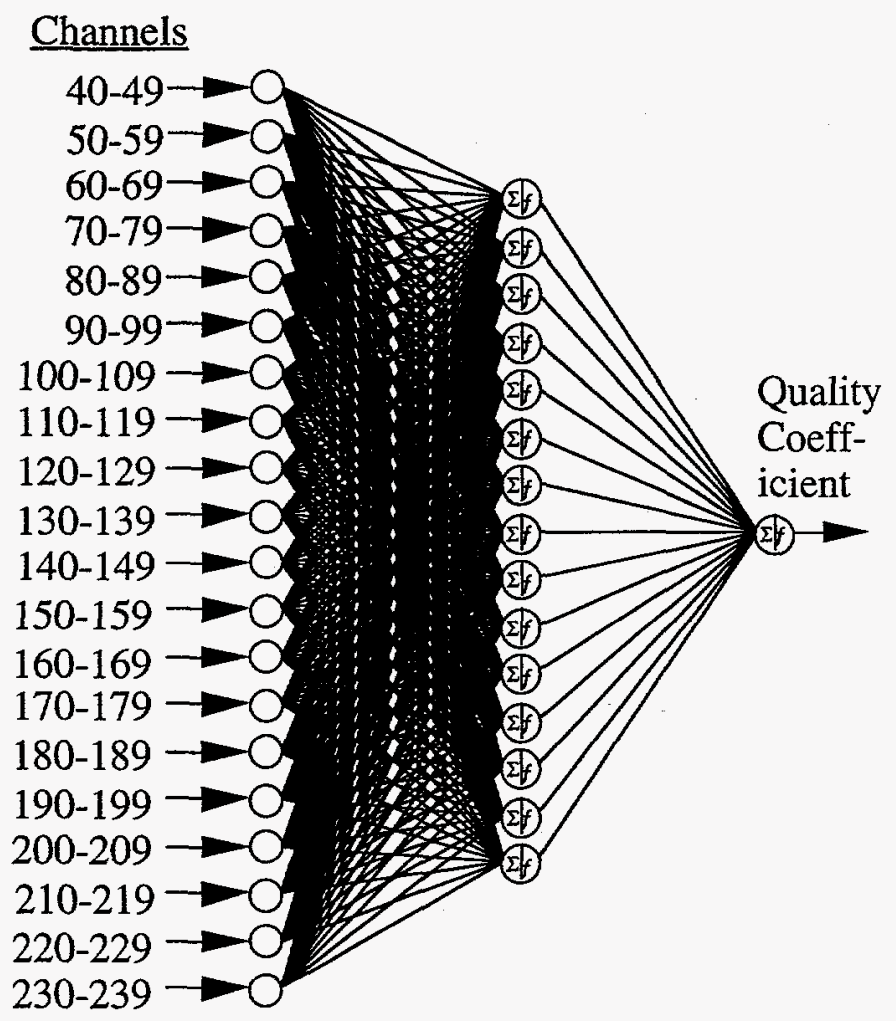

Fig 3. Artificial Neural Network (ANN) with 20 spectral values as input and one output which is the assessed quality factor for the spectra being analyzed. The figure shows that only the values from channels 40 to 239 of the 256 channel spectra are input to the ANN.

\section{RESULTS AND DISCUSSION}

The performance of an ANN is evaluated with testing data. An unbiased evaluation requires that these testing data are separate from the data used in training. In order to test all spectra available for this investigation, we partitioned the spectra into four equal sized sets and used the $\mathrm{K}$-fold testing approach. Each set was tested using a separate ANN that was trained with the other three sets. By using four ANNs and rotating the set used for testing, all spectra in the four testing sets were labeled. The K-fold testing approach resulted in an RMS error of 1.252 (MSE $=1.567$ ) measured over all data. The data set included one data point that exhibited a significant error. This data point was labeled 10 by the expert but only 3 by the ANN. A second examination of the point showed that the spectra of that data point was normal within the range of channels of interest. The expert acknowledged that this data point should have been labeled closer to the ANN assessed label. The removal of this data point from the testing set reduced the RMS error to 1.094 (MSE $=1.197$ ).

Our investigation shows that the expert knowledge of spectral quality can be transferred to an ANN system. The histogram in Figure 4 shows that only a small fraction of the spectra on hand have significant errors. Most spectra were qualified by the ANN system to be within \pm 2 coefficient values from those assigned by the expert. The deviation is within \pm 1 for $83.5 \%$ of the spectra and within \pm 2 for $97.1 \%$.

It is further noted that where extreme deviations existed, information in the spectra were not included in the expert's factor assignment. In particular, the worst mismatch had good spectral quality but included an additional isotope signal not in the majority of training spectra. Thus, it is expected that inclusion of a second condition for indicating the presence of the interfering isotope would improve the variance while providing an indicator of isotopic presence as an important side benefit.

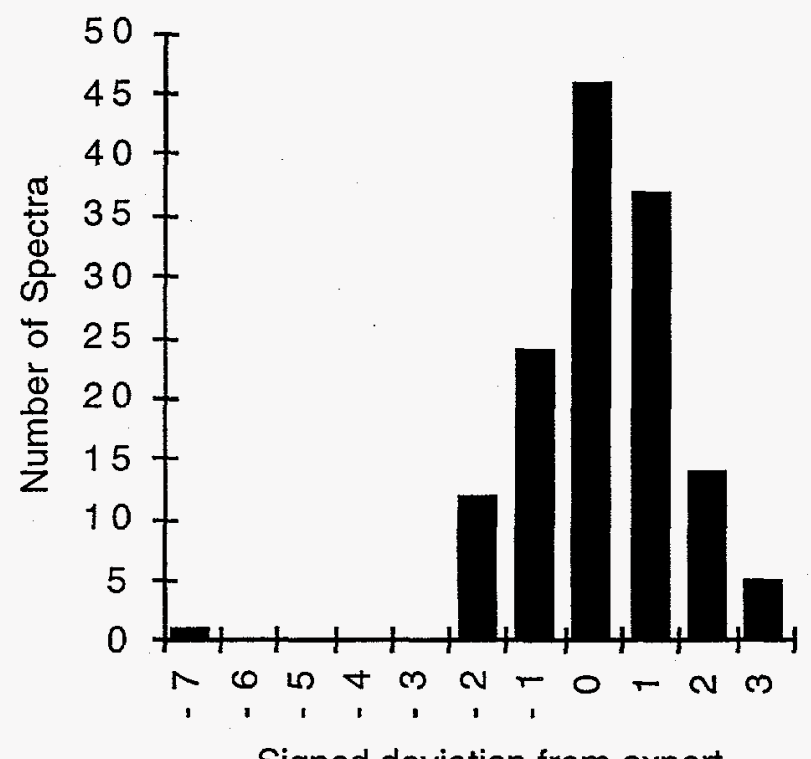

Fig 4. Distribution of deviation from expert. The figure shows the signed deviation (ANN - Expert) of the ANN assessed quality coefficients from the expert's coefficients.

\section{CONCLUSTON}

This investigation shows that ANNs are beneficial in automating analysis of spectral data. Only integrated or low resolution data are required for inferring reasonable decisions about the quality of the data. The results indicate that the ANN based approach is ready for inclusion into an automatic system for assessing quality of alpha spectra. Because inference is possible with reduced resolution, the commonly limited resources of in-line process monitoring equipment should still accommodate the ANN approach. Both quality and isotope identification alarm systems appear feasible and practical.

Additional information on ANN developments at Pacific Northwest Laboratory is available in the World Wide Web (WWW) pages of the Environmental Molecular Sciences Laboratory, which is accessible through such WWW clients as NCSA Mosaic.

URL: http://www.emsl.pnl.gov:2080/docs/cie/neural/ 


\section{REFERENCES}

[1] D.L. Wanningman, R.R. Farrell, A.V. Rochini, G.L. Troyer, "Analysis of Alpha CAM Performance at the Waste Isolation Pilot Plant (WIPP)," in Health Physics Society 39th Annual Meeting, paper WAM-F6, San Francisco, CA, June 1994.

[2] G.L. Troyer, "Algorithms To Correct For Radon Progneny Interference," in Health Physics Society 37th Annual Meeting, paper WAM-EA, Columbus, OH, June 1992.

[3] M.K. Alam, S.L. Stanton, and G.A. Hebner, "Near-Infrared Spectroscopy and Neural Networks for Resin Identification." Spectroscopy, pp. 30-40, February 1994.

[4] J.M. Lerner and T. Lu, "Practical Neural Networks Aid Spectroscopic Analysis," Photonic Spectra, pp. 93-98, August 1993.

[5] H. Lohninger and F. Stancl, "Comparing the performance of neural networks to well-established methods of multivariate data analysis: the classification of mass spectral data," Fresenius Journal of Analytical Chemistry, 344, pp. 186-189, 1992.

[6] B.J. Wythoff, S.P. Levine, and S.A. Tomellini, "Spectral Peak Verification and Recognition Using a Multilayered Neural Network," Analytical Chemistry, pp. 2702-2709, 1990. 